\title{
Infectious Causation of Parkinsonism: A Review
}

\author{
Arti Gupta ${ }^{1}$, VenkatashivaReddy.B ${ }^{2}$, Sidharth Sekhar Mishra ${ }^{3}$ \\ 1, 2, 3 (Centre for Community Medicine, All India Institute of Medical Sciences, India)
}

\begin{abstract}
Objective: Review was done to study how H. Pylori contributes to idiopathic Parkinsonism was discovered. Method: I searched the electronic databases for studies related to Prevalence of $H$ pylori in Parkinson's disease (Epidemiological studies and recruitment figures for clinical trial) and H. Pylori eradication in Parkinson's disease Randomised controlled trials and clinical controlled trials (nonrandomised). Finding: Prevalence of H. Pylori in Parkinsonism patient varied. The case control studies were conducted by many authors. Taking controls as siblings and assessed exposure by seropositivity of the cases for $H$ Pylori. Two studies observed cohort effect in seropositivity of H. Pylori in PD. Studies done in mice reported increase in motor symptoms in mice infected with H. Pylori, similar to Parkinson. Randomised controlled trials have shown increased improvement in motor symptoms of Parkinson after eradication therapy of H. Pylori. One clinical trial also reported the same.

Conclusion: By series of study design in temporal progression it lead to discovery that H. Pylori plays a role in causation of Parkinsonism.
\end{abstract}

Key words: Helicobacter pylori, levodopa, Parkinson's disease

\section{Introduction}

Over the past two centuries, diseases have been separated into three categories: infectious diseases, genetic diseases, and diseases caused by too much or too little of some non infectious environmental constituent. At the end of the 19th century, the most rapid development was in the first of these categories; within three decades after the first cause-effect linkage of a bacterium to a disease, most of the bacterial causes of common acute infectious diseases had been identified. This rapid progress can be attributed to Koch's postulates rigorous systematic approach to identification of microbes as causes of disease. Koch's postulates were useful because they could generate conclusive evidence of infectious causation, particularly when the causative organisms could be isolated and experimentally transmitted and symptoms occurred soon after the onset of infection in a high proportion of infected individuals. While guiding researchers down one path, however, the postulates directed them away from alternative paths: researchers attempting to document infectious causation were guided away from diseases that had little chance of fulfilling the postulates, even though they might have been infectious.

During the past few decades, our understanding of human microorganism interactions changed fundamentally as we learned infectious agents can give rise to chronic illnesses. Parkinson's disease is a neurological disorder that kills dopamine-producing cells in some parts of the brain. People with the disease have trouble controlling their movements. It is estimated that 6.3 million people have Parkinson's worldwide, affecting all races and cultures. The age of onset is usually over 60 , but it is estimated that one in ten are diagnosed before the age of 50 , with slightly more men than women affected. ${ }^{1} \mathrm{H}$. pylori, gram negative , micro aerophilic bacteria is a common infection of the gut and is often associated with duodenal and gastric ulcers. ${ }^{2} \mathrm{Up}$ to $50 \%$ of the world's population harbor H. pylori in their upper gastrointestinal tract. ${ }^{3}$ More than infection is at work the host genetic makeup certainly plays an important role in determining whether an infection, even when mild or asymptomatic, leads to ulcers or any number of other chronic illnesses. To study how H. Pylori contributes to idiopathic Parkinsonism was discovered.

\section{Method}

We searched the following electronic databases: MEDLINE, PUBMED, Cochrane, BMJ, LANCET and Google Scholar for studies related to prevalence of $\mathrm{H}$ pylori in Parkinson's disease and $\mathrm{H}$ pylori eradication in PD. All databases were searched form inception and searches were updated on 31.march.2012. In addition, we checked reference lists of reviews and retrieved articles for additional studies. We searched International Conferences of Parkinson Disease and Movement Disorders (years2000 to 2012) for additional relevant articles. From the searches we reviewed the title and abstract of each paper and retrieved potentially relevant references. We extracted details of the study design, participant characteristics, study setting, intervention, and outcome data including details of outcome assessment. Studies identified to determine the prevalence of H pylori in Parkinson's disease and Randomised controlled trials and clinical controlled trials (nonrandomised) were not formally assessed for methodological quality. We report information on the prevalence of H. pylori in Parkinson disease descriptively as it was not feasible to pool the data from individual Randomised controlled trials and 
clinical controlled trials 1 studies. Stratified analyses were not possible due to the small number of studies included in the review.

\section{Results}

Searching the electronic databases yielded 114 references. Studies with 19 free full texts were included in the study. Role of H Pylori in Parkinson's disease was suspected long back in $1965 .{ }^{4}$ In early sixties physicians noted a correlation between stomach ulcers and Parkinson's disease and observed an excess of previously diagnosed peptic ulcer in IP. At that time, Helicobacter pylori had not yet been discovered and ulcers were believed to result from stress. ${ }^{8}$ Later in the 1980 's, H Pylori was shown to cause more than $90 \%$ of gastric ulcers. $^{5}$

In 1996 a case control study found that in parkinsonism, there is a higher prevalence of gastrointestinal ulcers than in age- and sex-matched controls or than in the reported rates for the general population. ${ }^{6}$ Another study in 1999 quantified the facets of parkinsonism in 33 elderly subjects with idiopathic parkinsonism and in their 39 siblings with double the number of controls and Specific-IgG antibody was assayed. The study showed Siblings, compared with controls, had hypokinesia of gait $(\mathrm{P}<$ or $=0.002)$, bradykinesia of hands $(\mathrm{P}=0.01)$, abnormal posture $(\mathrm{P}=0.001)$, rigidity $(\mathrm{P}<0.001)$. Both parkinsonians and siblings differed from controls in the odds of being $\mathrm{H}$. pylori seropositive. ${ }^{7}$ In 2000 stated eradication of a trigger has potential to change the approach to parkinsonism just as it did to peptic ulcer. ${ }^{8}$

Another study in same year examined serum H pylori anti-urease-IgG antibody levels in 105 patients with idiopathic Parkinson's disease and 210 without Parkinson's disease. Overall they found $48 \%$ of patients with Parkinson have to be seropositive and $40 \%$ of the controls. The authors found patients with Parkinson's disease were more likely to be seropositive $(\mathrm{P}<0.04)$ before 72.5 years of age. Controls showed a birth-cohort effect. ${ }^{9}$ A study in 2001 report that the area under the curve of L-dopa plasma concentration, following the administration of a single $250 \mathrm{mg} \mathrm{L}$-dopa dose, is augmented after $\mathrm{H}$. pylori eradication in Parkinson's disease patients. A prolongation of L-dopa clinical benefit was also observed. ${ }^{10}$ In 2003a study examined the prevalence of H. pylori infection in Parkinson's disease patients and of their spouses using 13C urea breath test. They found around 60 percent of Parkinson's patients were $\mathrm{H}$ pylori-positive. ${ }^{11}$ In 2005 a case control study present the first case-series evidence of efficacy of Helicobacter eradication, in parkinsonism advanced to the stage of cachexia. 12

In 2005 a randomised, placebo-controlled, double-blind efficacy study of $\mathrm{H}$ pylori eradication on the time course of the motor symptoms in Parkinson's disease was conducted. The authors found a significant increase in the estimated change in mean stride length among Parkinson patient with $\mathrm{H}$ pylori eradication compared to placebo, mean torque to flex and percentage body sway. ${ }^{13}$ Another study in 2005 found that eliminating infection in late parkinsonism can result in a cure. ${ }^{14}$ In 2006 a RCT was done in a group of H pylori infected patients with Parkinson's disease on levodopa mono therapy with motor fluctuations and revealed the similar results. ${ }^{15}$ Another RCT in 2006 of $\mathrm{H}$ pylori eradication found of the total more than one third Parkinson's disease Patients were H pylori-positive on serology and invasive procedure. ${ }^{16}$ In 2007 a doubleblinded RCT stated that the treatment of H. Pylori in patients with Parkinson's disease had shown improvement in clinical response to standard doses of levodopa. ${ }^{17}$ In 2008 a study using the urea breath test, demonstrated that $\mathrm{H}$ Pylori infection could interfere with the absorption of L-dopa and provoke motor fluctuations. H Pylori eradication can improve the motor fluctuations of $\mathrm{H}$ Pylori infected patients with PD. ${ }^{18}$

In 2008 was explicated that in PD, H. pylori antibodies discriminate for persistently abnormal bowel function, and specific abnormal duodenal enterocyte mitochondrial morphology. ${ }^{19}$ A study reported motor score/disease duration of $\mathrm{PD}$ was worse in the $\mathrm{H}$ pylori infected group than the non-infected group $(\mathrm{P}=$ 0.04). ${ }^{20}$ In 2010 a study presented data which demonstrates for the first time the ability of L-DOPA to influence the in vitro growth of $\mathrm{H}$. pylori in an iron-restricted minimal medium. ${ }^{21}$ In 2011 a study in mice revealed middle-aged mice infected with the ulcer-causing bacterium developed abnormal movement patterns over several months of infection. ${ }^{2}$

\section{Discussion}

Very few studies met the inclusion criteria for this review. Estimates in Patients with Parkinson's from the four studies identified from our searching range from $37 \%$ to $59 \% .^{(11,15)}$ Starting of identification of the cause was with a mere observation that PD patients were frequently having peptic ulcer. This was non scientific grounds and lacks the validity. This lead to a hypothesis further tested by case control studies of various authors. Most of these reported that both PD patients and their siblings differed from controls in H. pylori seropositiviting and symptoms. Birth cohort effect was seen as significant rise of antibody levels against $\mathrm{H}$. Pylori in patient with PD compared to siblings. Later randomized control trial where started to prove stronger association. The trials showed significant improvements in levodopa absorption and motor symptoms with $\mathrm{H}$ 
pylori eradication. Recently studies were conducted in mice to find out the causation. Results from an ongoing trial will add to this evidence base

\section{Conclusion}

By series of study design in temporal progression it lead to discovery that $\mathrm{H}$. pylori play a role in causation of Parkinsonism. H. Pylori full fill the Koch's postulate in case of PD. To establish the role of a infectious organism in a chronic disease this is the usual pathway followed by researchers. At present no recommendations can be made regarding widespread screening and eradication of $\mathrm{H}$. pylori infection PD patients. The potential benefits of $\mathrm{H}$ pylori eradication need to be balanced against the costs of screening and treatment. In this review few studies were included which can lead to bias. Having review on role other infectious disease agents in chronic diseases will provide and insight to young researcher in field of epidemiology.

\section{Acknowledgements}

We would like to thank Archana Gupta and Shailabh Sahu for enlightening \& guiding us with the topic.

\section{Abbreviation}

Parkinson disease (PD), Idiopathic parkinson (IP), Randomized control trial (RCT), Helicobacter pylori (H. Pylori)

\section{References}

[1] European Parkinson disease association 2011. Available at http://www.epda.eu.com/en/parkinsons/ (citated on 4. April.2012)

[2] K. Rees , R. Stowe, S. Patel, N. Ives , K. Breen, C. Clarke, and Y. Ben-Shlomo, Helicobacter pylori eradication for Parkinson's disease, Cochrane Database of Systematic.2011

[3] K. McColl, Helicobacter pylori infection, N Engl J Med, 362, 2010, 1597-604

[4] RR. Strang, The association of gastro-duodenal ulceration with Parkinson's disease, Med J Austr, 52, $1965,842-3$.

[5] RJ. Dobbs, Role of chronic infection and inflammation in the gastrointestinal tract in the etiology and pathogenesis of idiopathic parkinsonism, Part 1: eradication of Helicobacter in the cachexia of idiopathic parkinsonism. Helicobacter, 10(4), 2005, 267-75.

[6] E. Altschuler, Gastric Helicobacter pylori infection as a cause of idiopathic Parkinson disease and non-arteric anterior optic ischemic neuropathy, Med Hypotheses, 47(5), 1996, 413-4.

[7] A. Charlett, Parkinsonism: siblings share Helicobacter pylori seropositivity and facets of syndrome,. Acta Neurol Scand, 99(1), 1999, 26-35

[8] SM. Dobbs, Link between Helicobacter pylori infection and idiopathic parkinsonism, Med Hypotheses 55(2), 2000, 93-8

[9] RJ. Dobbs, Parkinsonism: differential age-trend in Helicobacter pylori antibody, Alimentary Pharmacology and Therapeutics, 14(9), 2000, 1199-205.

[10] M. Pierantozzi, Reduced L-dopa absorption and increased clinical fluctuations in Helicobacter pylori-infected Parkinson's disease patients, Neurol Sci, 22(1), 2001, 89-91.

[11] D. Wlodarek, Pylori and its influence on Parkinson's disease. Polski Merkuriusz Lekarski, 15(89), 2003, 428-31.

[12] RJ. Dobbs, . Role of chronic infection and inflammation in the gastrointestinal tract in the etiology and pathogenesis of idiopathic parkinsonism, Part 1: eradication of Helicobacter in the cachexia of idiopathic parkinsonism, Helicobacter, 10(4), 2005, 267-75

[13] IT. Bjarnason, Role of chronic infection and inflammation in the gastrointestinal tract in the etiology and pathogenesis of idiopathic parkinsonism. Part 2: response of facets of clinical idiopathic parkinsonism to Helicobacter pylori eradication. Helicobacter, 10(4), 2005, 276-87

[14] C. Weller, .Role of inflammation in gastrointestinal tract in aetiology and pathogenesis of idiopathic parkinsonism,. FEMS Immunol Med Microbiol, 44(2), 2005, 129-35

[15] M. Pierantozzi , Helicobacter pylori eradication and 1-dopaabsorption in patients with PD and motor fluctuations, Neurology, 66(12), 2006, 1824-9

[16] M. Pierantozzi , Reduced L-dopa absorption and increased clinical fluctuations in Helicobacter pylori-infected Parkinson's disease patients. Neurol Sci., 22(1), 2001, 89-91.

[17] Helicobacter Pylori Eradication and Motor Fluctuations in Parkinson's Disease Clinical Intervention Awards 2007

[18] WY. Lee, Helicobacter pylori infection and motor fluctuations in patients with Parkinson's disease, Mov Disord., 23(12), 2008, 1696-700

[19] RJ. Dobbs, Helicobacter hypothesis for idiopathic parkinsonism: before and beyond, Helicobacter, 13(5), 2008, 309-22

[20] Y. Tsuboi and T. Yamada, Prevalence of Helicobacter pylori infection amng patients with Parkinson's disease impact on clinical manifestations, Movement Disorders, 23(1), 2008

[21] M. Lyte , Microbial endocrinology as a basis for improved L-DOPA bioavailability in Parkinson's patients treated for Helicobacter pylori, Med Hypotheses, 74(5), 2010, 895-7

[22] TH. Saey, Mouse study finds stomach ulcer-causing microbes may also affect brain, ScienceDaily, 179(13), 2011 